\title{
Performance Requirements for the Phase-2 Tracker Upgrades for ATLAS and CMS
}

\author{
Duccio Abbaneo ${ }^{1,2, a}$ \\ ${ }^{1}$ CERN, Geneva, Switzerland \\ ${ }^{2}$ For the ATLAS and CMS Collaborations
}

\begin{abstract}
The High-Luminosity operation of the LHC poses unprecedented challenges for the design of the upgraded trackers of ATLAS [1] and CMS [2]. The stringent requirements imposed by the high particle density and integrated fluence reduce the phase-space of valid technical solutions, inducing both collaborations to design "all-silicon" trackers. On the other hand constraints and requirements coming for the rest of the detector lead to some different choices, especially for the outer trackers. The main requirements for the two tracking systems are reviewed, discussing the implications for the detector designs and layout, and explaining why some of the technical choices remain different in the two experiments. To conclude, some expected performance figures for the two tracking systems are presented.
\end{abstract}

\section{Requirements for the High-Luminosity LHC tracking systems}

\subsection{Requirements from higher particle rates}

A major challenge for the design of the HL-LHC trackers is the target integrated luminosity of $3000 \mathrm{fb}^{-1}$, which translates to unprecedented requirements in terms of radiation tolerance, particularly difficult to meet for sensors and on-detector electronics. The outer trackers will have to operate with unspoiled performance throughout the high luminosity program, planned for the decade 2026 - 2035, while it is conceivable that the inner parts of the pixel detectors could be replaced during a long shutdown, if the radiation tolerance requirements cannot be fulfilled.

ATLAS and CMS have performed detailed FLUKA [3] simulations to estimate the radiation exposure of the different detector regions, obtaining consistent results (Figure 1). The estimated levels are about one order of magnitude higher compared to the requirements that were used for the design of the existing trackers [4-7], reaching levels of $10^{16}$ particles per $\mathrm{cm}^{2}$ in the innermost pixel regions. The particle fluence depends essentially on $r$ (the distance from the beam axis), while the variation with $z$ (the distance from the nominal average collision point, along the beam axis) is very moderate.

When translating such radiation levels into requirements for the design of the tracking subdetectors, it should be noted that the boundary between the outer tracker and the pixel detector is located at a radius of about $350 \mathrm{~mm}$ in ATLAS and $200 \mathrm{~mm}$ in CMS. Furthermore, in the present tracker upgrade layouts the first pixel layer is located at $39 \mathrm{~mm}$ in ATLAS and at $29 \mathrm{~mm}$ in CMS, which translates to

a e-mail: Duccio.Abbaneo@cern.ch 

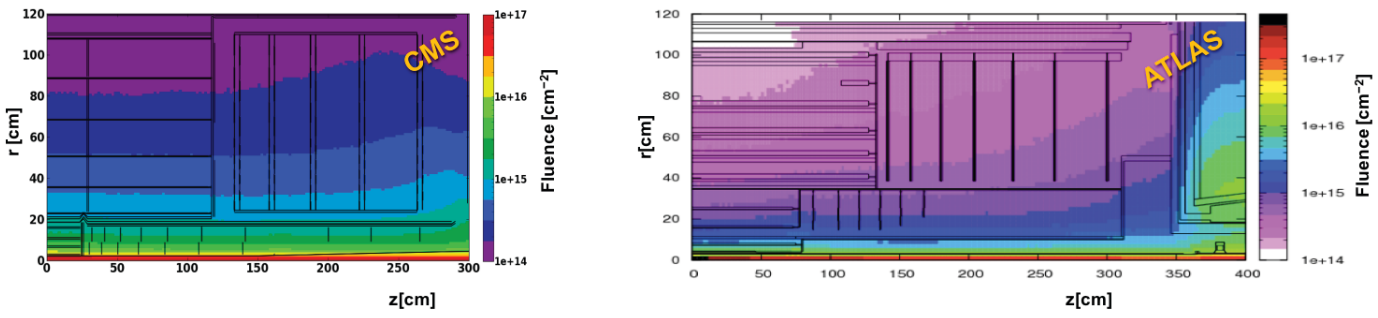

Figure 1. Integrated particle fluence in $1 \mathrm{MeV}$ neutrons equivalent per $\mathrm{cm}^{2}$, for the CMS [8] and ATLAS [9] phase- 2 trackers. The estimates shown correspond to a total luminosity of $3000 \mathrm{fb}^{-1}$ of $14 \mathrm{TeV}$ pp collisions.

nearly a factor of two difference in hit rates and integrated particle fluence per unit area. Some of the reasons for these differences will be discussed below.

The other basic requirement for the high-luminosity operation is the ability to maintain efficient tracking in a high-density particle environment. The experience with the present detectors shows that efficient tracking with low fake rate can be achieved if the channel occupancy does not exceed the $1 \%$ level. ATLAS and CMS are designing their trackers to operate with up to 200 pile-up collisions per bunch crossing, which requires a granularity between 5 and 10 times higher than that of the present trackers.

\subsection{Requirements from first-level trigger}

Besides the fundamental requirements directly linked to the increased particle rates in the tracking detectors, ATLAS and CMS also need to improve their first level trigger decision ${ }^{1}$, in order to fully profit from the higher luminosity. The enhancement of the trigger performance involves both a higher output rate of interesting events and an improved discriminating power of the event selection, which is more challenging in high pileup. Improved discriminating power will be achieved by using more information for the trigger decision, with a longer latency available for its processing. ATLAS and CMS plan to enhance the first level trigger rate and latency to the figures shown in Table 1, which are the new requirements for front-end electronics of the tracking systems.

In addition to the increased trigger rate and latency, CMS has chosen to also provide tracking information for the first-level trigger decision, implying that the tracker will send out some information at every bunch crossing. The different design choice is to some extent motivated by different boundary conditions in the two detectors (ATLAS has higher-granularity information from the calorimeters in the first-level trigger, while CMS has a stronger magnetic field, which helps for the specific implementation chosen), and has in turn led to several differences in the design of the upgraded trackers.

Table 1. Planned increase of output rate and latency for the first-level trigger decision in ATLAS and CMS.

\begin{tabular}{l|rll}
\hline \multirow{2}{*}{ ATLAS } & $100 \mathrm{kHz}$ & $\longrightarrow$ & $1000 \mathrm{kHz}$ \\
& $2.5 \mu \mathrm{s}$ & $\longrightarrow$ & $6.0 \mu \mathrm{s}$ \\
\hline \multirow{2}{*}{$\mathrm{CMS}$} & $100 \mathrm{kHz}$ & $\longrightarrow$ & $750 \mathrm{kHz}$ \\
& $3.2 \mu \mathrm{s}$ & $\longrightarrow$ & $12.8 \mu \mathrm{s}$ \\
\hline
\end{tabular}

\footnotetext{
${ }^{1}$ N.B.: The first-level trigger is called Level-0 in ATLAS and Level-1 in CMS.
} 

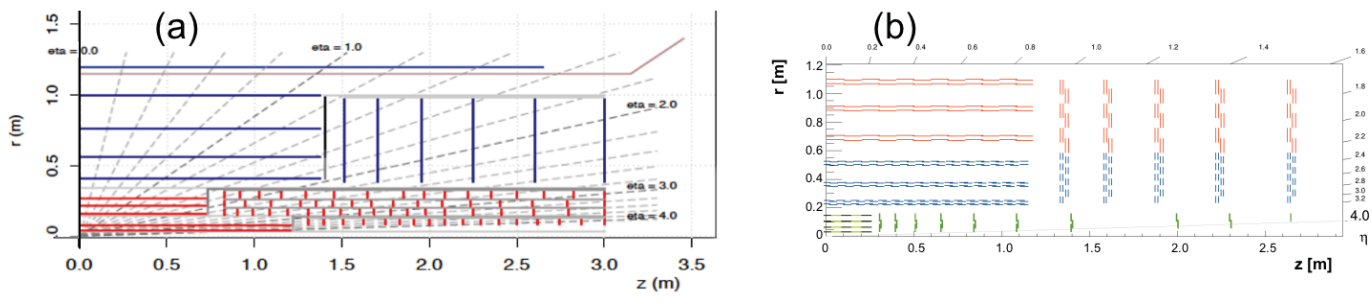

Figure 2. Possible layout of the ATLAS (a) and CMS (b) upgraded tracking systems. In the ATLAS tracker the boundary between outer tracker and pixel detector is located at about $350 \mathrm{~mm}$, while in CMS it is located at $200 \mathrm{~mm}$.

\subsection{Other improvements}

Both ATLAS and CMS are planning to extend the rapidity acceptance of their tracking systems up to about $|\eta|=4$. Tracking in the very forward region allows to assign forward jets to primary vertices, which improves performance in vector boson fusion and vector boson scattering physics. The rapidity extension concerns mostly the layout of the pixel detectors, with relevant implications on the detector mechanics and arrangement of the services.

The material in the tracking volume is a major limitation for the performance of the present detectors, affecting not only tracking but also the global event reconstruction. Reducing the amount of material in the upgraded trackers is a vital goal, although the requirements of highly enhanced functionalities mentioned above make such goal challenging to achieve.

ATLAS and CMS are still optimising the layouts of the tracking systems that will have to fulfill the requirements discussed. Two of the layouts under consideration are shown in Figure 2. For the CMS outer tracker, the layout shown is the baseline presented in the upgrade Technical Proposal [8], the collaboration is however also studying a different option with progressively tilted modules in the inner barrel layers. The layouts of the pixel detectors are still in the speculative phase for both collaborations.

\section{Outer trackers}

\subsection{Tracker input to first-level trigger in CMS}

The CMS tracker will provide information at each bunch crossing, in order to contribute to the firstlevel trigger decision. Such functionality relies upon local data reduction in the front-end electronics, in order to limit the volume of data that are sent out at $40 \mathrm{MHz}$. This is achieved with modules that are capable of rejecting signals from particles below a certain $p_{\mathrm{T}}$ threshold, referred to as " $p_{\mathrm{T}}$ modules" [10-12]. The modules are composed by two closely-spaced sensors read out by a common set of front-end ASICs, that correlate the signals in the two sensors and select the pairs ("stubs") compatible with particles above the chosen $p_{\mathrm{T}}$ threshold (Figure 3). A threshold of around $2 \mathrm{GeV}$ corresponds to a data volume reduction of roughly one order of magnitude, which is sufficient to enable transmission of the stubs at $40 \mathrm{MHz}$, while all other signals are stored in the front-end pipelines and read out when a trigger signal is received.

The $p_{\mathrm{T}}$ module concept relies on the fact that the strips of both sensors are parallel to the $z$-axis in the barrel and nearly radial in the end-cap, preventing the use of stereo strips to measure the $z$ 

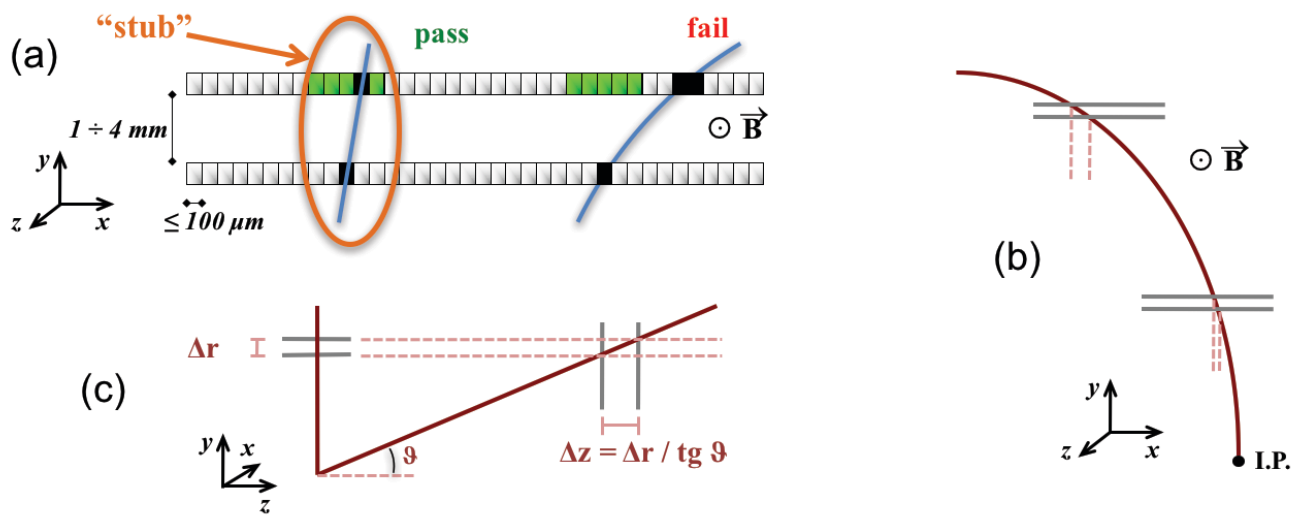

Figure 3. (a) Correlation of signals in closely-spaced sensors enables rejection of low- $p_{\mathrm{T}}$ particles; the channels shown in light green represent the "selection window" to define an accepted "stub". (b) The same transverse momentum corresponds to a larger distance between the two signals at large radii for a given sensor spacing. (c) For the end-cap disks, a larger spacing between the sensors is needed to achieve the same discriminating power as in the barrel at the same radius. The acceptance window can therefore be tuned along with a few different values of sensor spacing to achieve the desired $p_{\mathrm{T}}$ filtering in different regions of the detector. For a realistic pitch of about $100 \mu \mathrm{m}$, sufficient $p_{\mathrm{T}}$ resolution can be achieved down to a radius of about $200 \mathrm{~mm}$, thanks to the $3.8 \mathrm{~T}$ magnetic field of CMS.

coordinate. For this reason two versions of $p_{\mathrm{T}}$ modules have been realized: 2-Strip (2S) modules and Pixel-Strip (PS) modules (Figure 4). In PS modules one of the two sensors is segmented into macro-pixels of about $1.5 \mathrm{~mm}$ length, providing also a precise measurement of the $z$ coordinate. The main parameters of $2 \mathrm{~S}$ and PS modules are summarized in Table 2. The choice of pitch of $90 \mu \mathrm{m}$ and $100 \mu \mathrm{m}$ are driven by limitations in the line density on the hybrid for the $2 \mathrm{~S}$ module, and on the bump density of the C4 technology for the PS module. PS modules are deployed in the first three layers of the outer tracker, in the radial region of 200-600 mm (blue in the sketch of Figure 2b), while $2 \mathrm{~S}$ modules are deployed in the outermost three layers, in the radial region above $600 \mathrm{~mm}$ (red in the sketch of Figure 2b). The $z$ coordinates provided by the three PS layers enable some level of primary vertex discrimination for trigger tracks that do not have pixel coordinates, hence the interest to extend the range of PS layers towards low radii, up to the limit where the stub $p_{\mathrm{T}}$ resolution remains acceptable.

Table 2. Main parameters of the $2 \mathrm{~S}$ module and the PS module of the CMS tracker.

\begin{tabular}{c|c}
\hline 2S module & PS module \\
\hline \hline$\sim 2 \times 90 \mathrm{~cm}^{2}$ active area & $\sim 2 \times 45 \mathrm{~cm}^{2}$ active area \\
\hline $2 \times 1016$ strips: $\sim 5 \mathrm{~cm} \times 90 \mu \mathrm{m}$ & $2 \times 960$ strips: $\sim 2.5 \mathrm{~cm} \times 100 \mu \mathrm{m}$ \\
$2 \times 1016$ strips: $\sim 5 \mathrm{~cm} \times 90 \mu \mathrm{m}$ & $32 \times 960$ pixels: $\sim 1.5 \mathrm{~mm} \times 100 \mu \mathrm{m}$ \\
\hline Power $\sim 5 \mathrm{~W}$ & Power $\sim 8 \mathrm{~W}$ \\
\hline
\end{tabular}




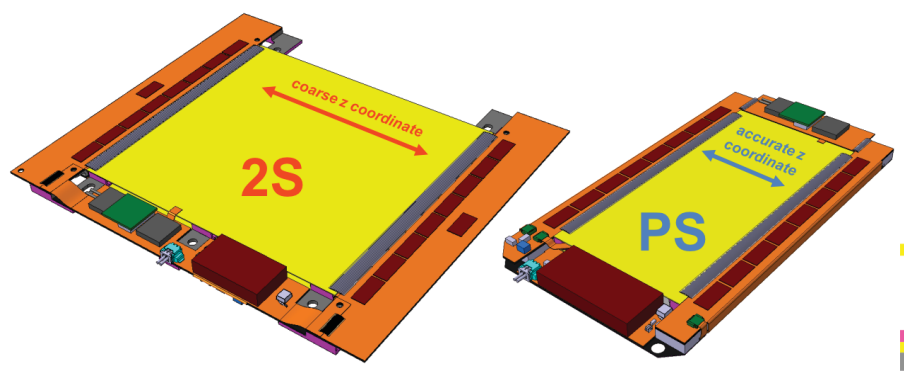

Figure 4. Silicon modules for the CMS tracker. 2S modules are composed of two strip sensors superimposed, PS modules of one strip sensor and one pixelated sensors. In the $2 \mathrm{~S}$ module, the sensors are read out from the sides, where high-density flex hybrids bring the signals from both sensors to a common set of readout ASICs (CBC, CMS Binary Chip), as shown in the rightmost sketch. In PS modules, one of the two sensors is pixelated, and read out by a dedicated Macro-pixel ASIC (MPA). Also in this case a flex hybrid provides the connectivity between the MPA and the Short-Strip ASIC (SSA), reading out the strip sensor.

\subsection{Modules and structures in ATLAS}

For the barrel region, ATLAS has designed modules with similar strip lengths as CMS (48 mm and $24 \mathrm{~mm}$ for the outer and inner layers, respectively), but could afford a smaller pitch of $75 \mu \mathrm{m}$, since the readout electronics collects signals from one sensor only. The readout hybrid is located on top of the sensor (Figure 5a), which avoids large inactive area at the edge of the modules, that can therefore be abutted on the same surface with minimal inefficiency in between.

Barrel layers are composed of "staves" (Figure 5c), each carrying two rows of modules on the two sides. Top and bottom modules have the strips tilted by $\pm 20 \mathrm{mrad}$ with respect to the $z$-axis, hence providing also a precise measurement of the $z$ coordinate.

In the end-caps, modules are arranged on "petals" (Figure 5b) that follow the same concept as the barrel staves. Several different sensor designs and variants of hybrid circuits are needed to cover the wedge-shaped surface with abutted modules. The granularity is similar to the barrel, with a typical pitch of $75-80 \mu \mathrm{m}$ and strip lengths of $20-30 \mathrm{~mm}$ in the inner rings, and $40-60 \mathrm{~mm}$ in the outer rings. On one side of the petal modules have radial strips, providing the $\phi$ coordinate, while on the other side the strips are tilted by $40 \mathrm{mrad}$, hence measuring also $r$.

\subsection{Modules and structures in CMS}

The $p_{\mathrm{T}}$ modules discussed in Section 2.1 populate the entire volume of the CMS outer tracker. The two halves of each module are read out independently by the hybrids on the two sides, which prevents the reconstruction of stubs when particles cross the module near the center, with a large incident angle (see sketch of Figure 6b). In a flat barrel layout (Figure 6a) such an effect translates into a geometrical inefficiency of stub finding which is larger than $30 \%$ at the edge of the first barrel layer. To overcome this limitation, CMS is studying an alternative layout where the first three barrel layers, that are populated by PS modules, feature progressively tilted modules, nearly perpendicular to incident particles over the entire barrel length (Figure 6c). The design of the mechanical structure for the tilted section is ongoing (Figure $6 \mathrm{~d}$ ) and the option appears to be promising. 


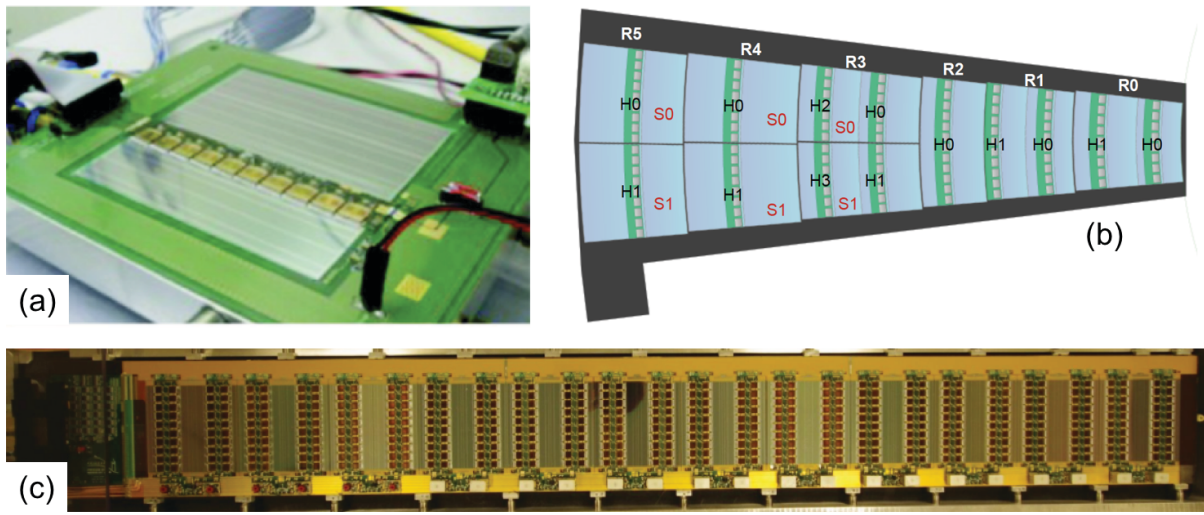

Figure 5. ATLAS silicon modules feature the readout hybrid integrated on top of the sensors, hence avoiding inactive area on the sides (a). In the barrel staves (c), two rows of modules are abutted on each side of the mechanical structure. A similar concept is implemented in the end-cap petals, where different sensor and hybrid versions are needed to cover the wedge-shaped surface.

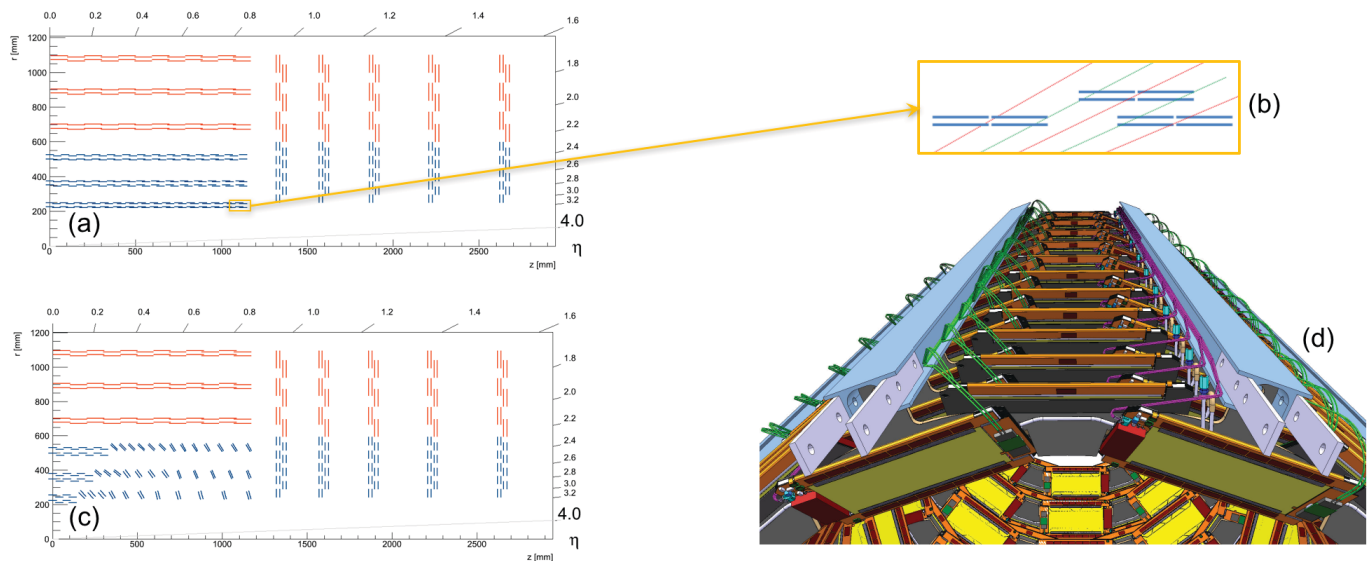

Figure 6. In the flat barrel layout (a) PS modules at the edge of the inner barrel layers have a large geometrical inefficiency for on-board stub finding (b): while the inefficiency at the edge of the modules can be compensated by implementing large overlaps between consecutive modules in $z$, the inefficiency in the center is irrecoverable (indicated by the red tracks). To overcome the problem, an alternative layout with progressively tilted modules (c) is under study. The design of the mechanics for the tilted layout is well advanced (d).

In the outer three layers of the barrel the effect of stub finding inefficiency is much less severe because the incidence angle of particles is less pronounced, the sensor spacing at those radii is smaller, and the modules are twice as long on the $z$ side. Modules are mounted on "ladders", where overlap in the $z$ view is achieved by placing the modules at different radii (Figure 7a). Along $\phi$, consecutive ladders are again staggered in $r$, hence a hermetic barrel layer is formed by modules located at four different radii. 


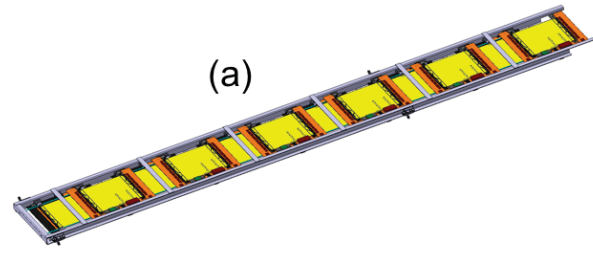

(b)

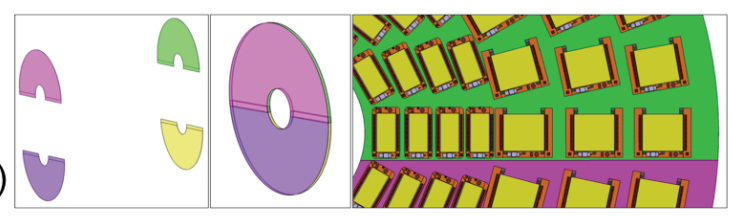

Figure 7. In the ladders of the CMS outer barrel (a), modules are mounted at two different radii in order to implement $z$ overlap. In the end-caps, the same rectangular modules are arranged on the four surfaces of a double-disk structure (b).

In the end-cap, due to the large radial extension (from $200 \mathrm{~mm}$ to nearly $1100 \mathrm{~mm}$ ) and the small size of PS modules, the use of wedge-shaped modules would lead to an impractical large number of sensor and hybrid varieties. Therefore the same rectangular modules used in the barrel are arranged on a double-disk structure: modules located at a given radius are mounted alternately on the two sides of a disk with overlap in $\phi$, forming a hermetic ring; consecutive rings along $r$ are mounted on different disks, hence also achieving radial overlap. Similarly to the barrel, a hermetic disk surface is achieved with modules mounted at four different $z$ locations.

\section{Pixel detectors}

The high-luminosity operation implies extreme challenges for the design of the pixel detectors in terms of radiation tolerance of sensors and readout electronics, as well as data volume to be stored in the front-end pipelines and sent out at high trigger rates. In order to cope with the reduced charge in the sensors, a readout chip with small cell size and low detector threshold is required. ATLAS and CMS are carrying out a common development in the framework of RD53 [13], to design a pixel chip with $2500 \mu \mathrm{m}^{2}$ cell size, in $65 \mathrm{~nm}$ CMOS technology.

Despite the common development for the front-end ASIC, some relevant differences remain in the layout of the two pixel detectors, in their present stage of development. Besides the different outer boundary discussed above, motivated by the fact that CMS implements macro-pixels in the intermediate radial range, in the current designs the inner boundary is also different, with the CMS pixel detector featuring a first layer at $29 \mathrm{~mm}$ average radius, while the ATLAS detector has the first layer at $39 \mathrm{~mm}$. The difference is quite relevant as it translates to nearly a factor of two difference in hit rates and particle fluence per unit surface. The difference is partially justified by the fact that the CMS pixel detetctor can be installed and extracted with the beam pipe in place, and is removed in the occasion of a beam pipe bakeout; hence it can be located as close as allowed by the mechanical tolerances. In comparison, in ATLAS the first layer of the pixel detector is mounted directly onto the beam pipe, which requires a certain thickness of insulation material for the beam pipe bakeout. CMS maintains as its design target to have a first layer at about $30 \mathrm{~mm}$ also for the high luminosity operation, while ATLAS is exploring the possibility of reducing the radius compared to the current design.

Another topic under study is the optimal pixel aspect ratio, for the assumed pixel surface of $2500 \mu \mathrm{m}^{2}$. CMS has studied the case of the most challenging barrel layer 1, comparing the $r-\phi$ and $z$ resolutions obtained on the single hit, for different assumptions on sensors thickness and chip detection threshold. The results, summarized in the plots of Figure 8, indicate that, notably in the 

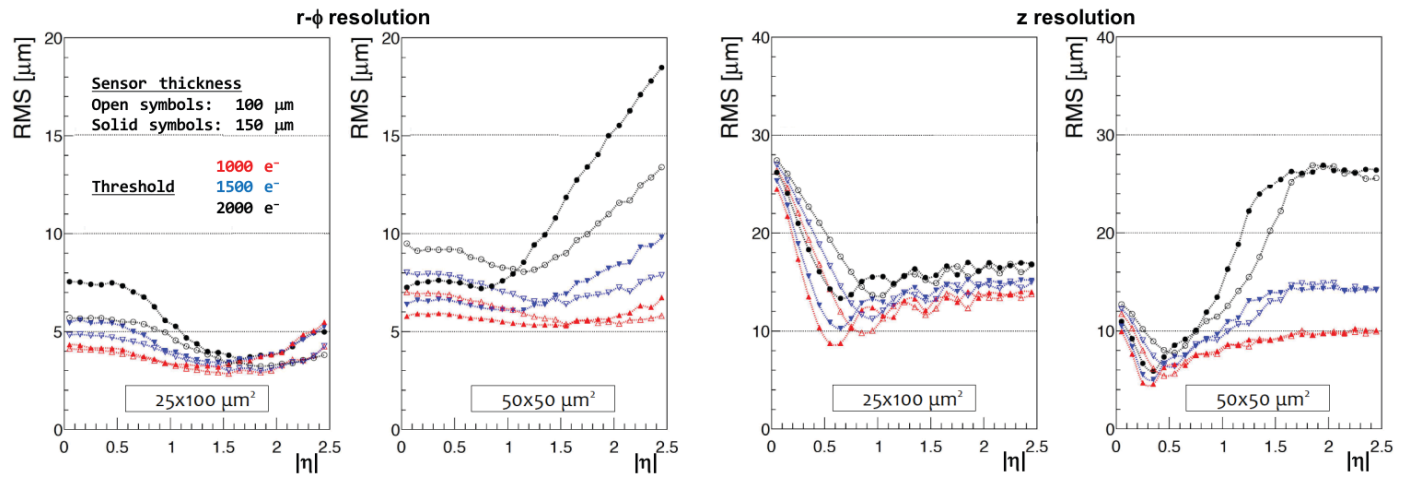

Figure 8. Comparison of single hit resolutions in $r-\phi$ and $z$ obtained in the first pixel barrel layer with square and rectangular pixels, for different assumptions on sensor thickness and detection threshold. Open and solid markers correspond to $100 \mu \mathrm{m}$ and $150 \mu \mathrm{m}$ silicon thickness, respectively; red markers correspond to a threshold of $1000 \mathrm{e}^{-}$, blue markers $1500 \mathrm{e}^{-}$and black markers $2000 \mathrm{e}^{-}$. Square pixels offer better $z$ resolution in the central region, while rectangular pixels give in general better performance in the other cases, and are less demanding on the chip threshold. These results are obtained with the flat barrel geometry of Figure 2b, and the simulation corresponds to unirradiated sensors.

forward part of the barrel, rectangular pixels of $25 \times 100 \mu \mathrm{m}^{2}$ offer better resolution, while being less demanding in terms of detection threshold and required bandwidth (which is particularly relevant for a first layer located at a radius of $30 \mathrm{~mm}$ ). ATLAS instead is focussing on the use of square pixels studying how to exploit the correlation between cluster length and track incident angle: details on these studies can be found in [14].

Concerning the overall layout, CMS has so far used the geometry shown in Figure $2 b$, derived from the "phase-1" detector with added disks to extend the rapidity coverage. It is however known that modifications to this geometry will be needed, because the detector insertion mechanism requires a larger opening in the forward part, beyond $z \approx 1500 \mathrm{~mm}$. The boundary between pixel detector and outer tracker will have to be moved to a higher radius in that region.

ATLAS has several pixel detector concepts under study. As an alternative to the flat barrel layout, two different options implementing progressively tilted modules are investigated, and could be deployed in some or all of the layers, as shown in the sketches of Figure 9. The design of both versions of the tilted geometry is well advanced, with realistic prototypes already built (Figure 10).

\section{Expected performance}

Both collaborations have produced performance estimates for the tracker concepts under study. In Figure 11 the efficiency as a function of transverse momentum is shown for the ATLAS tracker, along with the muon $p_{\mathrm{T}}$ resolution and the fraction of mis-reconstructed tracks. The solid black markers correspond to the baseline layout, offering good performance up to $|\eta|<4$, while the other markers correspond to cost-saving options with reduced coverage.

In Figure 12 the expected performance of the CMS upgraded tracker at 140 pileup is compared to the "phase-1" detector at 50 pileup. The upgraded tracker provides equivalent or better performance in the higher pileup conditions within the common acceptance range, while extending the acceptance 

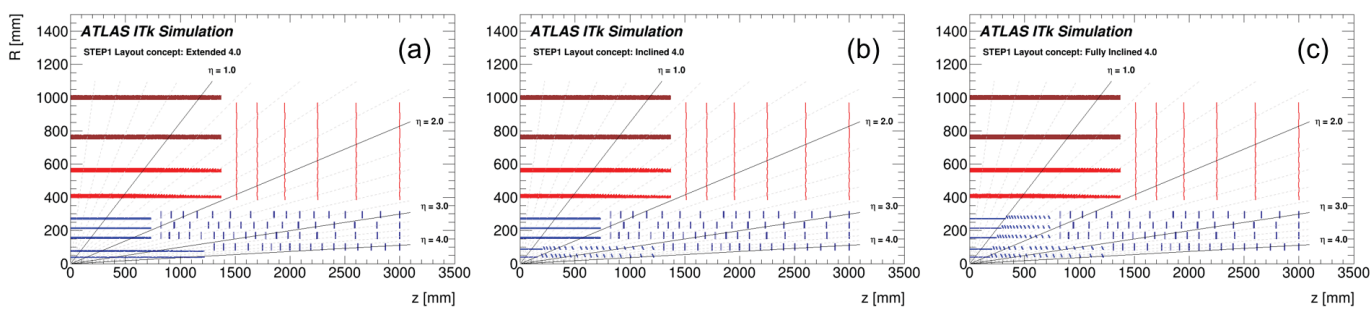

Figure 9. Layout of the ATLAS tracker with three different options for the pixel barrel: a geometry with flat layers (a), an option where the first two layers implement progressively tilted modules (b), and a third version with tilted geometry in all layers (c).
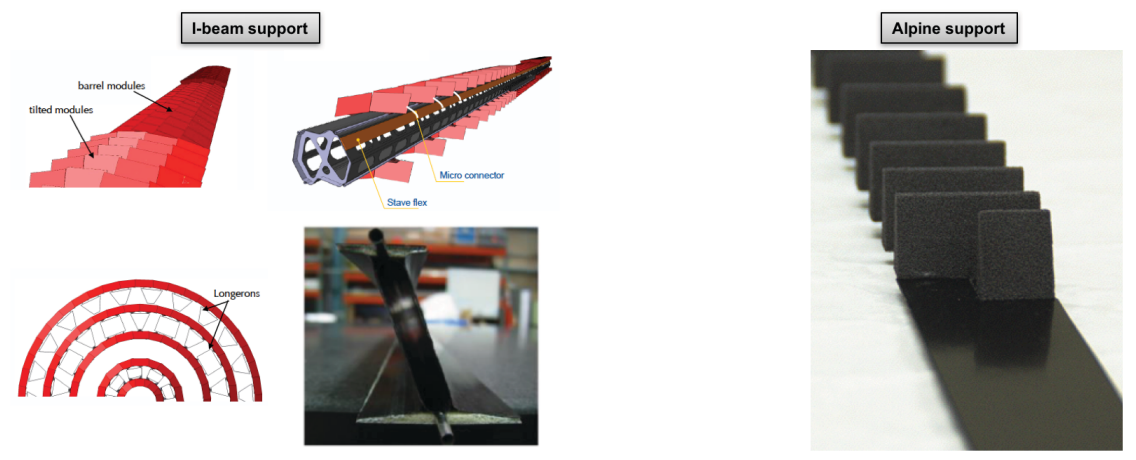

Figure 10. Two different options are being developed in ATLAS [15] to implement tilted modules in pixel barrel layers. In the I-beam concept (left) mechanical supports are shared between neighbouring layers, offering the possibility to realize lightweight structures of high stiffness, particularly suitable for long layers. The Alpine concept (right) is a straightforward adaptation of the flat stave concept, where carbon-fiber wedges are added to support modules with the desired tilt angle.

up to nearly $|\eta|=4$. The amount of material of the outer tracker is reduced by about a factor of two compared to the present tracker, despite the highly enhanced functionalities.

Another important aspect for the CMS tracker is the performance in the reconstruction of tracks for the first-level trigger, which was the subject of other contributions to this workshop [16].

\section{Conclusions}

The high-luminosity operation of the LHC entails unprecedented requirements in terms of particle density and integrated fluence for the trackers of ATLAS and CMS. Such requirements limit the phase-space of valid technical solutions, inducing both Collaborations to design "all-silicon" trackers.

The challenge is particularly difficult for the pixel detectors, where ATLAS and CMS are carrying out a common development for the readout chip, in the framework of RD53.

CMS has chosen to implement tracking in the first-level trigger decision, which has driven several specific design choices, especially in the outer tracker: the implementation of $p_{\mathrm{T}}$ modules limits the achievable pitch and requires the development of macro-pixels to measure the $z$ coordinate; the 

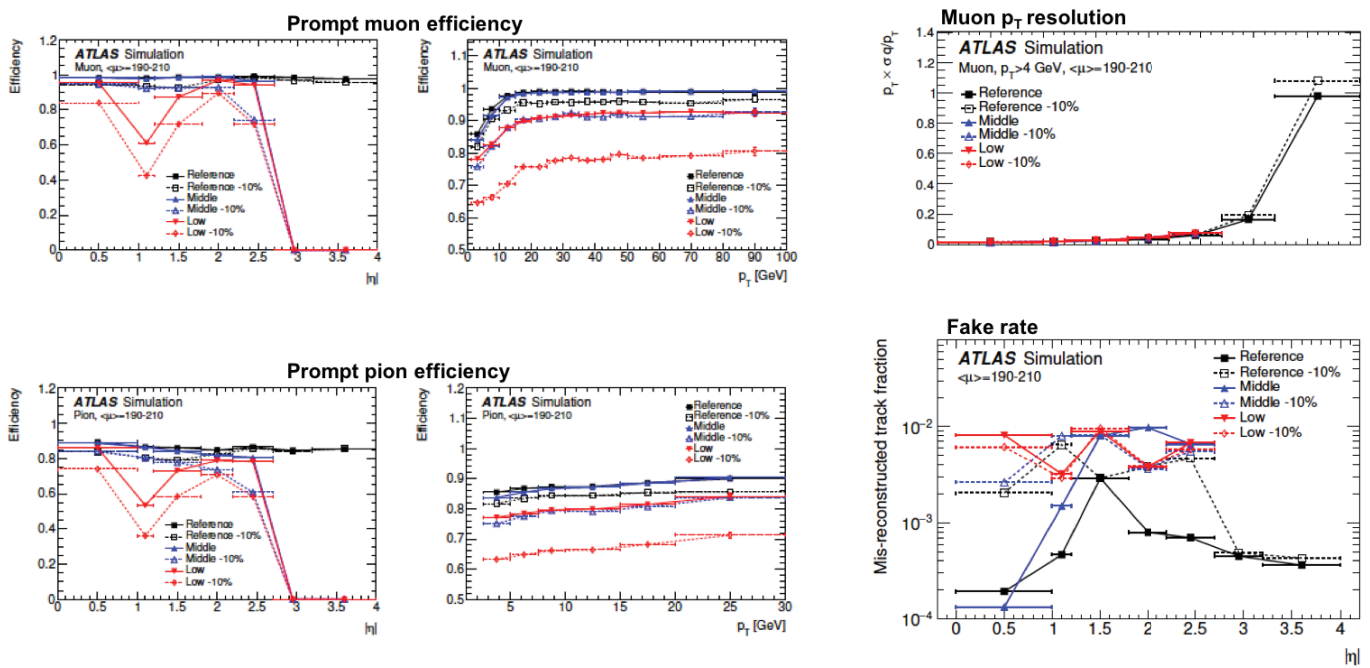

Figure 11. Main performance figures for the upgraded ATLAS tracker [15]. The baseline layout, corresponding to the solid black markers, provides excellent tracking performance up to $|\eta|=4$. The other markers correspond to various cost-saving options with reduced coverage.

boundary between outer tracker and pixel detector is pushed to lower radii to achieve primary vertex discrimination in trigger tracks; the large radial extension and half-size PS module disfavour the development of wedge-shaped modules for the end-cap; the need of mitigating the geometrical inefficiency for stub finding has motivated the development of the tilted layout for the inner barrel. The sketch of Figure 13 illustrates the cascade of implications.

The available performance estimates indicate that both detectors will provide efficient tracking in high pileup conditions, and at the same time offer improved resolution and extended acceptance.

While the design of the outer trackers is relatively advanced, layout options for the pixel detectors are still being explored, and further developments are underway. 

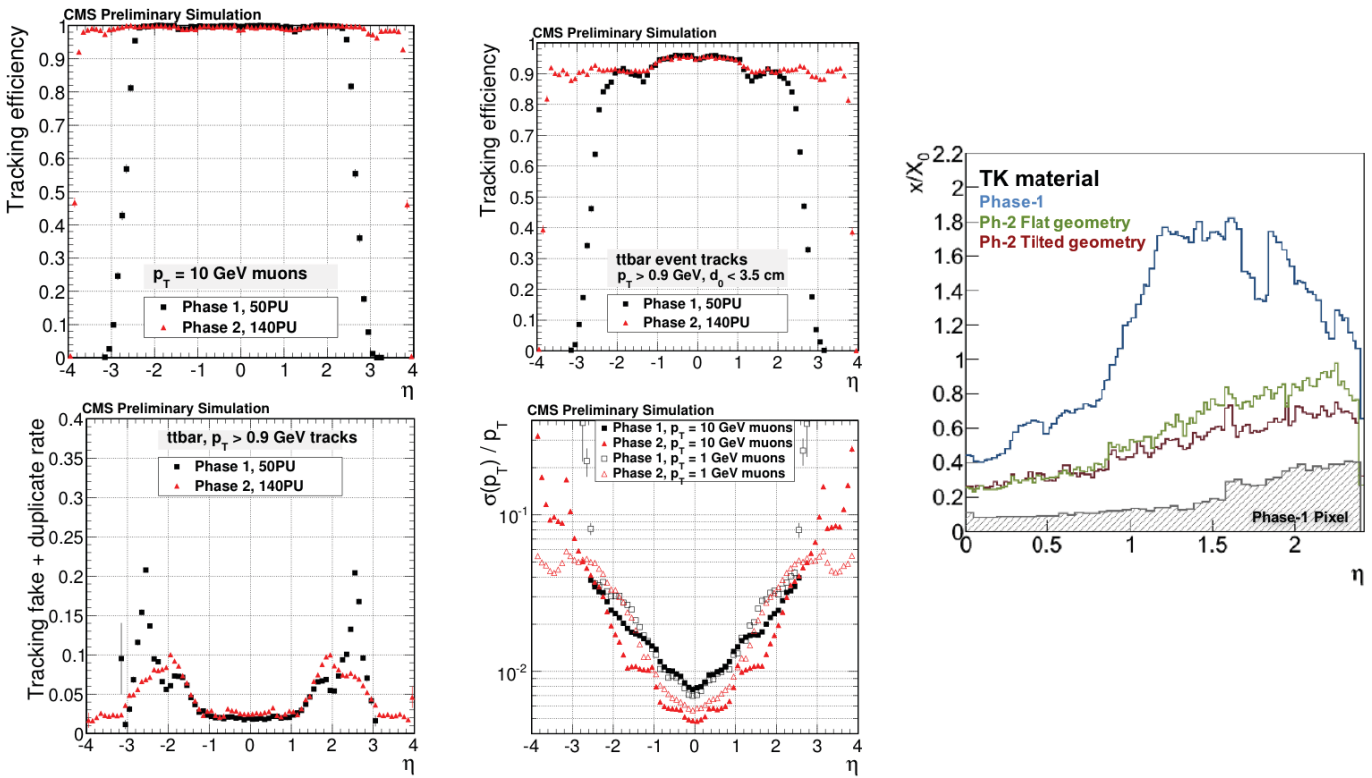

Figure 12. The performance of the upgraded CMS tracker in 140 pileup events is compared to the phase-1 tracker in 50 pileup events [8]. The upgraded tracker provides similar tracking efficiency and better resolution and fake rate, plus extended acceptance. The amount of material in the outer tracker is substantially reduced, and the tilted geometry for the inner barrel offers an additional reduction compared to the flat geometry. N.B.: A material estimate for the upgraded pixel detector is not yet available, therefore the phase-1 material distribution is used in this plot.

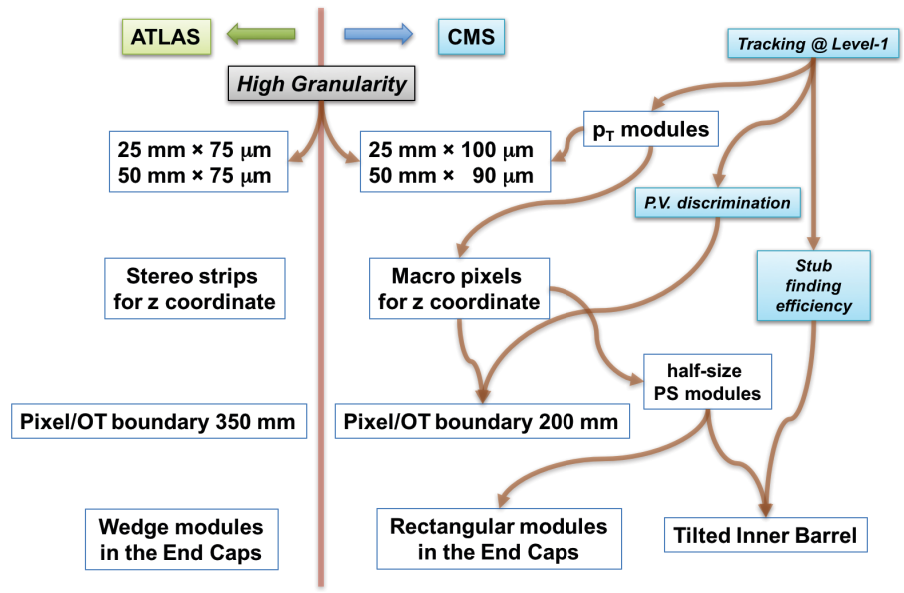

Figure 13. The sketch illustrates how the requirement of contributing tracking information to the first-level trigger decision for the CMS tracker has resulted in a cascade of different technical choices compared to the ATLAS tracker. 


\section{References}

[1] The ATLAS Collaboration, JINST 3, S08003 (2008), http: //stacks . iop.org/1748-0221/3/i=08/a=S08003

[2] The CMS Collaboration, JINST 3, S08004 (2008), http: //stacks. iop.org/1748-0221/3/i=08/a=S08004

[3] A. Ferrari, P.R. Sala, A. Fassò, J. Ranft, Tech. Rep. CERN-2005-010, CERN, Geneva, Switzerland (2005), https: //cds . cern. ch/record/898301

[4] The CMS Collaboration, Tech. Rep. CERN-LHCC-1998-006, CERN, Geneva, Switzerland (1998), https://cds. cern. ch/record/368412

[5] The CMS Collaboration, Tech. Rep. CERN-LHCC-2000-016, CERN, Geneva, Switzerland (2000), https://cds . cern. ch/record/490194

[6] The ATLAS Collaboration, Tech. Rep. CERN-LHCC-1997-016, CERN, Geneva, Switzerland (1997), https://cds. cern. ch/record/331063

[7] The ATLAS Collaboration, Tech. Rep. CERN-LHCC-1997-017, CERN, Geneva, Switzerland (1997), https: //cds . cern. ch/record/331064

[8] The CMS Collaboration, Tech. Rep. CERN-LHCC-2015-010, CERN, Geneva, Switzerland (2015), https://cds . cern. ch/record/2020886

[9] The ATLAS Collaboration, Tech. Rep. CERN-LHCC-2012-022, CERN, Geneva, Switzerland (2012), https: //cds . cern. ch/record/1502664

[10] C. Foudas, A. Rose, J. Jones, G. Hall (2005), https://arxiv.org/abs/physics/0510227

[11] G. Hall, M. Raymond, A. Rose, JINST 5, C07012 (2010), http://iopscience.iop.org/article/10.1088/1748-0221/9/10/C10034/meta

[12] M. Pesaresi, G. Hall, JINST 5, C08003 (2010), http://iopscience.iop.org/article/10.1088/1748-0221/5/08/C08003/meta

[13] J. Christiansen, M.L. Garcia-Sciveres (eds.), Tech. Rep. CERN-LHCC-2013-008, CERN, Geneva, Switzerland (2013), https: //cds . cern. ch/record/1553467

[14] S. Viel et al., Nucl. Inst. \& Meth. A 831, 254 (2016), http://www . sciencedirect.com/science/article/pii/S0168900216301346

[15] The ATLAS Collaboration, Tech. Rep. CERN-LHCC-2015-020, CERN, Geneva, Switzerland (2015), https://cds . cern. ch/record/2055248

[16] G. Fedi, these proceedings; L. Skinnari, these proceedings 